2. Normenkonflikte und kollektive Dynamiken digitaler Gesellschaften 


\section{Informationelle Privatheit, Standardautorität und soziale Pathologien ${ }^{1}$}

Wulf Loh

\section{Einleitung}

In den letzten Jahren hat die philosophische Theoriebildung auch im Bereich Privatsphäre/Privatheit einen practical turn erlebt. ${ }^{2}$ Diesem Verständnis nach wird Privatheit als Set von Normen in sozialen Praktiken beschrieben, das sich in den Rollenverpflichtungen der Praxisakteure niederschlägt. Damit eröffnet sich die Möglichkeit, „defective role performances and defective social relations" 3 mit Blick auf Privatheitsnormen in bestimmten Praktiken auszumachen. Gleichzeitig kaufen sich die Verfechterinnen eines solchen praxistheoretischen Ansatzes aber zumeist auch einen gewissen Praxispositivismus ein: Eine bestimmte Praxis gilt nach Nissenbaum dann als privat, wenn sie sich durch eine „contextual integrity“ auszeichnet, d. h. dass in ihr ein „appropriate flow of information“ sichergestellt ist. ${ }^{4}$ Was aber jeweils als ,appropriate، gilt, hängt von der jeweiligen Praxis $a b$ und wird auch in dieser entschieden. Damit ergibt sich für praxistheoretische Privatheitsmodelle die Schwierigkeit, dass sie alle Privatheitsarrangements prima facie als angemessen begreifen müssen, die in einer bestimmten Praxis auch als angemessen aufgefasst werden. Ein solcher Positivismus hat die unschöne Konsequenz, dass auch Praktiken, die wir unter keinen Umständen als privat bezeichnen wollten, privat sind, sofern die Akteure selbst die Privatheitsnormen für angemessen halten.

1 Mein Dank gilt den Veranstaltenden der Konferenz „Verantwortung in digitalen Kulturen" und den Herausgebenden dieses Sammelbandes. Sie haben verschiedene Versionen dieses Aufsatzes kritisch begleitet und konstruktiv verbessert. Speziell bedanken möchte ich mich bei Martin Hennig, dessen Kommentare sehr wertvoll waren, sowie ganz besonders Anne Deremetz, die den Aufsatz mehrfach akribisch gelesen und dabei mit ihren Nachfragen und Hinweisen immer zuverlässig ins Schwarze getroffen hat. Alle noch verbleibenden Fehler und Ungenauigkeiten sind meinem eigenen Versäumnis geschuldet.

2 Vgl. Nissenbaum (2010); Roessler/Mokrosinska (2013).

3 Roessler/Mokrosinska (2013: S. 780).

4 Nissenbaum (2010: S. 149). 
Um einige dieser Konsequenzen zu umgehen, habe ich bereits an anderer Stelle dieses praxistheoretische Standardmodell um eine sozialontologische Ebene ergänzt und zu einem sozialontologischen Anerkennungsmodell von Privatheit (SAMP) erweitert. ${ }^{5}$ Dieses Modell werde ich im Folgenden nutzen, um die Idee sozialer Pathologien der Privatheit zu motivieren. Kurz gesagt geht es bei dem SAMP darum, die normative Beschreibung der Praxis im Sinn von Normen und Rollen um eine zweite Hinsicht zu ergänzen, auf der sich die Praxisteilnehmenden als ,Standardautorität ${ }^{6}$ anerkennen müssen. ${ }^{6}$ Mit dieser Anerkennung ergeben sich weitreichendere Privilegien und Verpflichtungen über die jeweiligen eigenen Rollenverpflichtungen hinaus, auf die ich in Abschnitt 2.1 näher eingehe.

Die kontextuelle Integrität bzw. der adäquate Informationsfluss eines Verdatungskontextes wird nach diesem Modell genau dann sichergestellt, wenn innerhalb einer Verdatungspraxis alle Trägerinnen konstitutiver Rollen (TkR) - dies gilt besonders für die verdateten Subjekte - sich wechselseitig als Standardautorität anerkennen. Dabei wird das Kriterium der Standardautorität jedoch nicht einfach von außen an die Praxis herangetragen, sondern ergibt sich aus der normativen Struktur von sozialen Praktiken selbst: Weil in sozialen Praktiken Handlungsmuster internalisiert und habituell reproduziert werden, müssen diese im Fall divergierender Interpretationen thematisch werden können. Nur so sind die Sozialintegration und die Reproduktion sozialer Praktiken möglich.

Damit ist auch schon ein erster Hinweis darauf gegeben, wie sich diesem Modell zufolge, defective role performances and defective social relations' ausbuchstabieren lassen. ,Mangelhaft' bzw. ,defizitär'sind Rollenperformanzen und Sozialbeziehungen innerhalb einer Praxis $p$ dann, wenn hier nicht alle Trägerinnen konstitutiver Rollen als Standardautorität anerkannt werden. $\mathrm{Zu}$ Pathologien werden diese, wenn aus strukturellen Gründen bestimmten TkR die Anerkennung als Standardautorität verweigert wird. Sofern diese strukturelle Missachtung der Standardautorität in Verdatungspraktiken geschieht, handelt es sich um eine Pathologie informationeller Privatheit.

Spätestens dann, wenn diese Verweigerung innerhalb einer bestimmten Verdatungspraxis allgemein als soziotechnische Unumgänglichkeit akzeptiert und auf diese Weise essentialisiert wird, lässt sich von einer Pathologie

5 Vgl. Loh $(2018 ; 2019 b)$.

6 Vgl. zum Konzept der Standardautorität Stahl (2013: Kap. 5.5). Meine Verwendungsweise des Konzepts unterscheidet sich leicht von Titus Stahls ursprünglicher Idee. 
zweiter Ordnung sprechen. In diesem Fall steht die Verdatungspraxis den verdateten Akteuren wie ein Naturereignis gegenüber und verdeckt auf diese Weise, dass sie wie jede andere Praxis "gegeben wie gemacht" ${ }^{\text {"7 }}$ ist. Die sich daraus ergebenden psychomotivationalen Resignationen („Google hat ja sowieso alle meine Daten!“) äußern sich unter Umständen auch in kognitiven Dissonanzen wie dem oft untersuchten Privacy Paradox. ${ }^{8}$

Mithilfe eines solchen Modells sozialer Pathologien informationeller Privatheit können Verdatungspraktiken als normativ unangemessen ausgewiesen werden, ohne einen den Praktiken externen Maßstab der Kritik anlegen zu müssen. Vielmehr wird dieser Maßstab aus der Struktur sozialer Praktiken selbst - sowie der Überprüfung von Verdatungspraktiken an dieser Struktur - gewonnen. Dies werde ich in Abschnitt 2.1 noch genauer ausführen.

\section{Das sozialontologische Anerkennungsmodell von Privatheit}

\subsection{Standardautorität}

Für das Verständnis des sozialontologischen Anerkennungsmodells von Privatheit (SAMP) ist es sinnvoll, hier in aller Kürze die wichtigsten Merkmale des Begriffs einer sozialen Praxis in Erinnerung zu rufen. An anderer Stelle habe ich sechs grundlegende Aspekte des Praxisbegriffs herausgearbeitet, die meiner Ansicht nach jeweils notwendig und gemeinsam hinreichend für die Definition einer sozialen Praxis sind. ${ }^{9}$

Dieser Definition nach sind soziale Praktiken (1) wiederholte, aufeinander abgestimmte Handlungsmuster, die (2) bestimmten Regeln folgen, welche (3) zumindest zum Teil als „Knowing-Hows“10 internalisiert und routinemäßig befolgt werden. Diese Praxisregeln werden spätestens (4) in dem Moment thematisch, in dem die Verhaltenserwartungen einzelner Akteure aufgrund unterschiedlicher Regelinterpretationen unterlaufen werden und aufgrund dessen eine explizite Kritik an den Rollenperformanzen formuliert wird. Die wechselseitigen Verhaltenserwartungen werden also (5) in sozialen Rollen mit den dazugehörigen Rollenprivilegien

7 Jaeggi (2014: S. 120).

8 Vgl. Dienlin/Trepte (2015).

9 Vgl. Loh (2018, 2019b, 2019a: Kap. 2.1).

10 Vgl. Ryle (2009: Kap. 2). 
und -verpflichtungen „generalisiert"11. Zusätzlich zu diesen Rollenverpflichtungen verweist (6) die Tatsache konfligierender Interpretationen sei es in Form divergierender Performanzen, sei es in Form expliziter Kritik an den Performanzen Anderer - auf einen deontischen Status zweiter Ordnung, d. h. auf wechselseitige Verpflichtungen auf einer grundlegenderen normativen Ebene.

Dieser Status zweiter Ordnung ist für das SAMP entscheidend, da sich hier die Praxisteilnehmenden wechselseitig als „Standardautorität ${ }^{\text {"12 }}$ anerkennen. ${ }^{13}$ Dieser Status manifestiert sich wiederum in zwei Hinsichten, die häufig ineinander übergehen, sich aber analytisch unterscheiden lassen: Die erste Hinsicht betrifft die wechselseitige Rechenschaftsplicht der Praxisakteure untereinander für die Erfüllung ihrer jeweiligen Rollenverpflichtungen in der Praxis $p .{ }^{14}$ Indem sie sich wechselseitig als Standardautorität anerkennen, besitzen sie alle als Teilnehmende in $p$ die Autorität, sich wechselseitig mit Blick auf ihre jeweiligen Rollenverpflichtungen zu kritisieren. Umgekehrt bringen sie mit einer solchen Kritik zum Ausdruck, dass sie die Performanzen der anderen Akteure in deren jeweiligen Rollen als kompetente Versuche einer Interpretation dieser Rollen ansehen und sich diesbezüglich als Standardautorität anerkennen.

So macht die Kritik an einer Zugbegleiterin, die neben der Fahrkarte der Fahrgäste auch noch den Grund der Reise wissen will, zwar einerseits deutlich, dass die Fahrgäste dieses Rollenverhalten nicht als angemessen ansehen. Gleichzeitig wird ihr Verhalten dennoch als kompetenter Versuch der Rolleninterpretationen verstanden - und sie somit als Standardautorität anerkannt. Dies gilt jedoch nicht für die Passagierin, die plötzlich aufspringt und die Fahrkarten zu kontrollieren beginnt. Die geäußerte Kritik: „Was machen Sie denn da? Sie sind doch nicht die Schaffnerin!“, zeigt gleichzeitig an, dass ihr Verhalten eben nicht als kompetenter Interpretationsversuch der Zugbegleiterinnenrolle angesehen wird, sie somit nicht als Standardautorität anerkannt ist. ${ }^{15}$

Die wechselseitige Anerkennung als Standardautorität beinhaltet dieser ersten Hinsicht zufolge also über die jeweiligen Rollenverpflichtungen hi-

11 Mead (1934: S. 155).

12 Vgl. Stahl (2013: Kap. 5.5).

13 Der folgende Abschnitt orientiert sich an Loh (2019b: S. 40 f.).

14 Ich verwende die Begriffe Praxisteilnehmende, Praxisakteure und Praxismitglieder synonym. Alle so bezeichneten Handelnden haben eine konstitutive Rolle innerhalb dieser Praxis inne.

15 Dies gilt wohlgemerkt für die Rolle als Zugbegleitende, in der Rolle Fabrgast kann sie immer noch als Standardautorität anerkannt werden. 
naus eine Art Meta-Verpflichtung, auf mögliche Kritik an den performativen Interpretationen der eigenen Rolle zu antworten und diese Interpretationen im Hinblick auf die Normen von $p$ zu erläutern. In dieser ersten Hinsicht verweist der deontische Status zweiter Ordnung auf die allen Teilnehmenden innewohnende gleiche Autorität, sich wechselseitig in ihren Rollenperformanzen kritisieren zu können, wie gerade im Zugbeispiel gesehen.

Bei der zweiten Hinsicht hingegen stehen nicht mehr die Performanzen einzelner Rollenträgerinnen und deren Norminterpretationen im Mittelpunkt, sondern die Verteilung der Rollenprivilegien und -verpflichtungen innerhalb von $p$ selbst. Dazu gehört auch die Frage, welche Rollen überhaupt in $p$ vorkommen sollten. Um beim Zugbeispiel zu bleiben, fielen hierunter Auseinandersetzungen über Sinn und Unsinn von Zugbegleiterinnen, also die Frage, ob die Rolle der Zugbegleiterin überhaupt in der Praxis Zugfahren vorkommen sollte. Dagegen würde eine Kritik daran, dass Zugbegleiterinnen Fahrkarten kontrollieren dürfen, die Verteilung von Rollenprivilegien adressieren.

Die erste Hinsicht der wechselseitigen Anerkennung als Standardautorität betrifft also die Normbefolgung in $p$, d. h. die Rollenperformanzen und die "reactive attitudes" 16 der anderen Praxisteilnehmenden in Bezug auf diese. Die zweite Hinsicht dagegen zielt auf die Normsetzung in $p$, d. h. die vorkommenden Rollen und die jeweils dazugehörigen Rollenstatus. Wie aus den Beispielen deutlich wird, ist diese Unterscheidung analytischer Natur, da sie in der Praxis häufig verschwimmt. Die Kritik an einem bestimmten Rollenverhalten schlägt leicht in eine Kritik an den in $p$ anerkannten Rollenverpflichtungen und -verteilungen um. Umgekehrt entzünden sich Auseinandersetzungen über die normative Struktur von $p$ in vielen Fällen an den tatsächlichen Rolleninterpretationen einzelner Akteure in $p$.

Damit Praktiken also langfristig funktionsfähig bleiben und die Möglichkeit zu substanzieller Veränderung eröffnen, muss prinzipiell eine wechselseitige Kritik der Praxisteilnehmenden an den dortigen Handlungsvollzügen möglich sein. Dies betrifft wesentlich die erste Hinsicht der Standardautorität, auf der sich alle Praxisakteure wechselseitig die Möglichkeit von Kritik an ihren Handlungen als Rolleninterpretationen zuerkennen und gleichzeitig die Meta-Verpflichtung der Rechenschaftspflicht für diese Performanzen übernehmen.

16 Strawson (1962: S. 66). 
Wie aber sieht es mit der zweiten Hinsicht aus, also der Möglichkeit, an der Struktur der Praxis selbst sowie an der Verteilung der Rollenprivilegien und -verpflichtungen innerhalb von $p$ Kritik zu üben? Sind diese auch notwendig für das Funktionieren von $p$ ? Immerhin kann man sich eine ganze Reihe autoritärer Praktiken vorstellen, in denen jede Kritik an der Praxisstruktur von bestimmten Akteuren bzw. Rollenträgerinnen nicht vorgesehen ist und scharf geahndet wird. Hierauf lassen sich zwei Antworten geben:

Zum einen gehen, wie weiter oben schon angemerkt, die beiden Hinsichten ineinander über. Eine Kritik an der Struktur entzündet sich häufig an bestimmtem transgressiven Rollenverhalten. Eine Intervention mit Blick auf die erste Hinsicht kann also leicht auch die zweite betreffen. Wie wir aber gesehen haben, ist die Kritik der ersten Hinsicht sozialontologisch notwendig für die Reproduktion von sozialen Praktiken überhaupt. Das bedeutet, dass bestimmte autoritäre Praktiken über kurz oder lang defizitär oder gar pathologisch werden, wenn sie Kritik vonseiten bestimmter Akteursgruppen an dem Rollenverhalten anderer Akteure nicht mehr zulassen. Darauf werde ich im nächsten Abschnitt genauer eingehen.

Zum anderen ist eine wechselseitige Anerkennung als Standardautorität auch aus einer sozialpsychologischen Perspektive für die Sozialintegration wichtig. Um die Teilnehmenden langfristig und normativ zu binden, muss eine Praxis $p$ in der Lage sein, sie zu motivieren, $p$ aufrechtzuerhalten und durch ihre Rolleninterpretationen zu reproduzieren. Dies geschieht jedoch nur dann freiwillig, wenn die Teilnehmenden nicht nur ihre eigenen Rollen so interpretieren können, wie sie es während ihrer Sozialisation internalisiert haben, ${ }^{17}$ sondern auch $p$ als Ganzes. Wenn die wechselseitige Kritik am Rollenverhalten Anderer auf irgendeine Weise verunmöglicht wird, werden die Akteure $p$ früher oder später nicht mehr als ibre Praxis ansehen und nur so lange reproduzieren, als sie dazu gezwungen werden. Die normative Kraft von $p$ besteht dann nicht mehr aufgrund von internalisierten Praxisregeln, sondern aufgrund von externem Zwang. Eine solche Praxis wird nur als „modus vivendi“ ${ }^{18}$ reproduziert werden und verfällt, sobald der äußere Zwang entfällt.

Die gegenseitige Anerkennung als Standardautorität ist also einerseits notwendig, damit soziale Praktiken überhaupt bestehen und ihre sozialontologische Funktion im Sinne der Stabilisierung von Verhaltenserwartungen erfüllen können. Andererseits spielt sie, wie gerade gesehen, bei der

17 Vgl. Berger/Luckmann (1969).

18 Rawls (2002: S. 50). 
Sozialintegration eine entscheidende Rolle, da die Praxisakteure $p$ nur dann aufrechterhalten und reproduzieren werden, wenn sie der Überzeugung sind, dass dies die richtige Art und Weise ist, eine jeweilige Instanz von $p$ zu realisieren. Sobald jedoch ihre Interpretationen von $p$ keinen Einfluss mehr auf $p$ erhalten, da sie nicht als Standardautorität anerkannt sind, wird sich die Struktur von $p$ immer mehr von ihren Interpretationen entfernen.

Obwohl also das Konzept der Standardautorität zunächst einmal lediglich die Stabilitätsmechanismen von sozialen Praktiken auf sozialontologischer Ebene expliziert, beinhaltet dieses Konzept eine schwache Normativität. Dies liegt daran, dass die wechselseitige Anerkennung aller Akteure in sozialen Praktiken keine rein deskriptive Aussage über unsere soziale Welt darstellt, da sie faktisch in vielen Praktiken nicht oder nur unvollständig besteht. Vielmehr enthält sie auch ein (normatives) Element der Wünschbarkeit von Weltzuständen: Die Befolgung von Praxisregeln und die Reproduktion der Praxis aufgrund von Sozialisation und Norminternalisierung ist einem rein äußerlichen Zwang vorzuziehen, da letzterer die Stabilität einer Praxis nie garantieren kann.

Aus diesem sehr kurzen Blick auf die Ontologie sozialer Praktiken lässt sich nun gut ersehen, wer die Akteure einer Praxis sind, d. h. wem eine Standardautorität zukommen (muss): Standardautoritäten in $p$ sind alle Trägerinnen konstitutiver Rollen in $p$, d. h. derjenigen Rollen, ohne die sich $p$ nicht instanziieren ließe. So unterscheidet sich die Praxis des Schenkens in ihren jeweiligen Instanziierungen - und möglicherweise Subpraktiken zum Teil erheblich, aber um als Schenken gelten zu können, bedarf es mindestens der Rollen der Schenkenden und der Beschenkten. Im Gegensatz zu akzidentiellen Rollen, die zufällig in Form der Rolle der ,Zuschauenden $^{619}$ in $p$ vorkommen, individuieren konstitutive Rollen $p$ als diese bestimmte Praxis. ${ }^{20}$

Trägerinnen konstitutiver Rollen (TkR) müssen sich aus sozialontologischer Perspektive deshalb wechselseitig als Standardautoritäten anerkennen, weil sie diejenigen Akteure markieren, die innerhalb von $p$ dergestalt handeln, dass ihre Interpretationen die Praxis wirkmächtig reproduzieren

19 Vgl. Goffman (1969).

20 Vgl. Searle (1969: Kap. 2.II). Dabei ist die Individuation von Praktiken allein über ihre konstitutiven Rollen nicht hinreichend, wie schon das obige Beispiel des Zugfahrens zeigt (es gibt Regionalbahnen ohne Zugbegleiterinnen und Flughafen-Shuttles ohne Lokführerinnen). Vielmehr bedarf es hierzu eines Überlegungsgleichgewichts zwischen Praxisnormen, Rollen und Zielen. Vgl. Loh (2019a: Kap. 2.1.1). 
bzw. verändern. Sie sind letztlich die Akteure, die über ihre Sozialisation entscheidend die Praxis stabilisieren - und damit in einem sozialontologischen Sinn konstituieren.

\subsection{Privatheit}

Mithilfe dieser Spezifizierung der Praxisakteure über ihre Rollenfunktionen lässt sich nun Privatheit genauer für die in der Privatheitsdebatte grundlegenden Domänen dezisionaler und informationeller Privatheit spezifizieren. ${ }^{21}$ Dezisionale Privatheit bezieht sich hierbei vor allem auf die Freiheit individueller Lebensentscheidungen, d. h. auf die Möglichkeit, „vor unerwünschtem Zutritt im Sinne von unerwünschtem Hineinreden, von Fremdbestimmen bei Entscheidungen und Handlungen geschützt zu sein." 22 Der Inhalt dieser Freiheit wird von der jeweiligen Praxis bestimmt. Auf einer sozialontologischen Ebene ist mit dezisionaler Privatheit somit das Recht gemeint, Trägerinnen akzidentieller Rollen nicht als Standardautoritäten anerkennen zu müssen. Sie können die Praxis interpretieren und Kritik an dem Rollenverhalten der Trägerinnen konstitutiver Rollen üben, letztere sind jedoch nicht verpflichtet, sich mit Blick auf diese Kritik zu rechtfertigen (Standardautorität in erster Hinsicht).

Wichtiger jedoch für die vorliegende Untersuchung ist die Domäne informationeller Privatheit. Hiermit wird häufig entweder die Freiheit gemeint, persönliche Informationen preisgeben oder zurückhalten zu können (control), ${ }^{23}$ oder aber die Möglichkeit der Zugangsbeschränkung zu solchen Informationen (access). ${ }^{24}$ James Moor sowie Herman Tavani haben diese beiden Aspekte in ihrer „restricted access/limited control“-Theorie (RALC) zusammengefasst. ${ }^{25}$ Mit dieser doppelten Qualifizierung informationeller Privatheit lassen sich wiederum die beiden grundlegenden Datenschutzkonzepte abbilden: Zum einen der Selbstdatenschutz, bei dem das Individuum in die Lage versetzt wird, in einer wohlinformierten Entschei-

21 An anderer Stelle führe ich ausführlicher die verschiedenen in der Literatur zu findenden Domänen von Privatheit wie örtliche, physische, mentale, räumliche, assoziative, Zugangs- und Kontrollprivatheit auf die hier genannten grundlegenden Domänen dezisionaler und informationeller Privatheit zurück. Vgl. Loh (2018: S. 235-236).

22 Rössler (2001: S. 25).

23 Vgl. Westin (1967); Fried (1984).

24 Vgl. Gavison (1980); Allen (1988).

25 Vgl. Moor (1997); Tavani (2007). 
dung über die eigenen Daten zu verfügen (limited control), zum anderen der Systemdatenschutz, dem zufolge staatliche Organe den Zugriff auf persönliche Informationen beschränken, um beispielsweise das Grundrecht auf informationelle Selbstbestimmung zu schützen (restricted access).

Die Aspekte Kontrolle und Zugang verdeutlichen auch noch einmal, dass es bei informationeller Privatheit nicht um „natürliche“ (im Sinne von faktischer), sondern um „normative Privatheit“ ${ }^{26}$ geht. Privatheit misst sich also gerade nicht daran, ob auf bestimmte ,undocumented personal information" 27 einer Person von anderen tatsächlich zugegriffen wird oder nicht. Vielmehr geht es um die Legitimität dieses Zugangs zu solchen Informationen und die normativ eingeräumte Möglichkeit der Kontrolle darüber. Diese werden vom jeweiligen Kontext bestimmt, d. h. von den Normen der jeweiligen Praxis $p$, welche sich in den verschiedenen Rollenprivilegien und -verpflichtungen widerspiegeln.

Sofern also informationelle Privatheit normativ - und damit ist gemeint, als Verpflichtung in den Rollen von $p$ - institutionalisiert ist, müssen diejenigen Individuen, um deren persönliche Informationen es geht, auch als Standardautoritäten mit Blick auf diese Privatheitsnormen anerkannt werden. Dies deshalb, da sie unabhängig davon, ob sie eine konstitutive Rolle innerhalb von $p$ innehaben, als verdatete Subjekte notgedrungen TkR mit Blick auf die korrespondierende Verdatungspraxis $p_{v}$ sind. So handeln beispielsweise Individuen in einem videoüberwachten Zug nicht nur in ihrer Rolle als Fahrgäste (p), sondern auch in ihrer Rolle als verdatete Subjekte der Überwachungspraxis Videoüberwachung im Zug $\left(\mathrm{p}_{\mathrm{v}}\right)$. Da letztere sich ohne die Rolle der verdateten Subjekte nicht instanziieren ließe, sind diese notwendig $\mathrm{TkR}$ in $p_{v}$.

Nun lässt sich genauer bestimmen, was informationelle Privatheit nach dem SAMP bedeutet: Informationell privat ist eine Praxis p genau dann, wenn sich alle TkR in allen korrespondierenden Verdatungspraktiken $p_{v 1-n}$ wechselseitig als Standardautorität anerkennen. Dies gilt speziell für die Verdatungssubjekte von $p_{v 1-n}$, denen notwendig eine Standardautorität in diesen Praktiken zukommt. Als Grenzfall ist es also auch möglich, dass in einer bestimmten Verdatungspraxis alle TkR alles übereinander wissen (oder auch, dass nur einige alles von anderen wissen), solange dies von allen TkR in ihrer Eigenschaft als Standardautorität nicht bestritten wird.

26 Moor (1990: S. 77).

27 Parent (1983: S. 346). 


\section{Pathologien erster und zweiter Ordnung}

Aus dem zuvor Gesagten lässt sich nun eine vorläufige Antwort darauf geben, was eine Pathologie informationeller Privatheit ausmacht: Pathologisch können Verdatungspraktiken dann werden, wenn in ihnen Trägerinnen bestimmter Rollen bzw. Rollentypen aus strukturellen Gründen nicht als Standardautoritäten anerkannt werden, obwohl sie TkR der Verdatungspraxis $\mathrm{p}_{\mathrm{v}}$ sind. In diesem Fall werden, wie oben gesehen, einerseits die sozialontologischen Wirkmechanismen von sozialen Praktiken auf lange Sicht verunmöglicht, andererseits wird die Sozialintegration als eine der primären Funktionen sozialer Phänomene auf Dauer untergraben. Mit anderen Worten: Wenn sich in einer Praxis nicht alle TkR als Standardautoritäten anerkennen, wird die Praxis über kurz oder lang auf die eine oder andere Art und Weise defizitär.

Um diesen Mechanismus in seiner Auswirkung für informationelle Privatheit im Speziellen zu explizieren, werde ich im Folgenden in aller Kürze zwischen Pathologien erster und zweiter Ordnung unterscheiden. ${ }^{28}$ In einem zweiten Schritt werde ich einige Beispiele für defizitäre bzw. pathologische Verdatungspraktiken vorstellen, anhand derer sich paradigmatische Weisen der Pathologisierung von informationeller Privatheit ablesen lassen. Auch wenn soziale Pathologien oftmals als ,Krankheiten der Gesellschaft ${ }^{6}$ verstanden werden, ${ }^{29}$ sollen hier zunächst einmal einzelne pathologische Praktiken bzw. Bündel von Praktiken in den Blick genommen werden. Sofern sich daraus ein gesellschaftsweites oder lebensformübergreifendes Phänomen ergibt, besteht immer noch die Möglichkeit, die gewonnene Analyse zu skalieren.

Der Begriff der sozialen Pathologie impliziert eine „Vorstellung von Normalität“ ${ }^{\text {30 }}$, die sich - so die zu Beginn angesprochene praxispositivistische Sicht - kulturabhängig in sozialen Normen und Praktiken sedimentiert hat. In diesem Sinne ist Nissenbaums, contextual integrity‘ zu verste-

28 Vgl. Celikates (2009: Kap. III.3); Zurn (2011). An anderer Stelle habe ich dafür plädiert, zwischen drei Arten von Pathologien (Fehlentwicklungen, Pathologien erster und zweiter Ordnung) zu unterscheiden. Vgl. Loh (2017). Für die vorliegende Untersuchung hält die dort vorgenommene zusätzliche Unterscheidung jedoch keinen analytischen Mehrwert bereit, daher beschränke ich mich hier auf die gängige Differenzierung.

29 Vgl. Honneth (2014). Axel Honneth sieht das Konzept sozialer bzw. gesellschaftlicher Pathologien schon in Rousseaus „Abhandlung über den Ursprung und die Grundlagen der Ungleichheit unter den Menschen“ avant la lettre angelegt.

Honneth (2000: S. 57). 
hen: ${ }^{31}$ Was als „appropriate flow of information“32 in $p$ gelten muss, lässt sich letztendlich nur vor dem Hintergrund eines bestimmten, historisch kontingent gewachsenen Ethos verstehen. „Defective role performances and defective social relations" 33 werden dann als Inkonsistenzen innerhalb von $p$ bzw. als Inkohärenzen mit Blick auf dieses Ethos bestimmt. Auf diese Weise ist zwar eine ,interne Kritik' möglich, ${ }^{34}$ diese erschöpft sich aber notgedrungen im Aufzeigen interner Inkonsistenzen bzw. Inkohärenzen.

Dagegen geht das SAMP einen Schritt weiter: Es ermöglicht eine Pathologiediagnose auf sozialontologischer Ebene, indem es auf eine fehlende wechselseitige Anerkennung als Standardautorität abstellt. ${ }^{35}$ Damit werden nicht mehr die Normen von $p$ oder ein bestimmtes kultur- bzw. gesellschaftsspezifisches Ethos als Maßstab für die Bedingungen herangezogen, die den Praxis-, Kultur- oder Gesellschaftsmitgliedern eine „unverzerrte Form der Selbstverwirklichung erlauben" ${ }^{36}$. Vielmehr bemisst sich das Defizitäre einer Praxis daran, dass - wie schon mehrfach betont - mit der Verweigerung der Anerkennung als Standardautorität die sozialontologischen und sozialintegrativen Funktionen von sozialen Praktiken nur noch unzureichend erfüllt werden können. ${ }^{37}$

Nicht jede Verweigerung der Anerkennung als Standardautorität ist dabei jedoch gleich pathologisch. Hierfür müssen die Missachtungen struktureller Natur sein: Erstens dürfen sie nicht nur kontingent einzelne Rollenträgerinnen betreffen, sondern typischerweise alle Trägerinnen einer bestimmten Rolle (beispielsweise der Rolle Verdatungssubjekt, aber auch Geschlechterrollen o. ä.). ${ }^{38}$ Zweitens muss die Anerkennungsverweigerung

31 Vgl. Nissenbaum (2004, 2010).

32 Nissenbaum (2010: S. 149).

33 Roessler/Mokrosinska (2013: S. 780).

34 Vgl. Stahl (2013: Kap. 1.1); Jaeggi (2014: Kap. 5).

35 Titus Stahl fasst diese Form der Diagnose unter dem Begriff Verdinglichungskritik (2013: Kap. 8).

36 Honneth (2000: S. 57).

37 Stahl spricht in diesem Zusammenhang von einer sozialontologischen „Metakritik“, die „die Weise, in der nicht nur diese oder jene spezifische Norm, sondern Normativität überhaupt in einer Praxis mittels Anerkennungsbeziehungen institutionalisiert wird“, betrifft. Stahl (2013: S. 420).

38 Ich kann hier nicht klären, ob diskriminatorisch hochrelevante soziale Kategorisierungen wie Geschlecht, ethnische Zugehörigkeit, sexuelle Präferenz usw. sozialontologisch am sinnvollsten als Rollen, Identitäten oder Gruppen zu fassen sind. Vgl. hierzu auch Haslanger (2000, 2005). In Anlehnung an ein Verständnis von doing gender werde ich für die Belange dieses Aufsatzes davon ausgehen, dass sich diese sozialen Kategorien auch produktiv als Rollenidentitäten ausbuchstabieren lassen. Vgl. West/Zimmerman (1987). 
aufgrund der Struktur von $p$ bestehen. Hier lassen sich wiederum zwei Möglichkeiten unterscheiden:

In einem ersten Fall sind die von der Nichtanerkennung als Standardautorität betroffenen Praxisteilnehmende - oder zumindest die meisten von ihnen - noch in der Lage, die Missachtung zu erkennen und auch zu artikulieren. Die strukturelle Verhärtung einer solchen Pathologie erster Ordnung zeigt sich vor allem darin, dass die Einsprüche und Kritiken der Betroffenen systematisch - also andauernd und umfassend - ignoriert oder blockiert, bzw. ihr Anspruch als nicht-normativ oder gesellschaftlich irrelevant zurückgewiesen werden. Honneth macht in diesem Zusammenhang deutlich, dass

bereits die offizielle Auszeichnung als, soziale Bewegung' das Resultat eines Kampfes um Anerkennung ist, den untergründig die von sozialem Leid betroffenen Gruppen oder Individuen um die öffentliche Wahrnehmung und Registrierung ihrer Probleme geführt haben. ${ }^{39}$

Dabei sind sich die Betroffenen jedoch - das unterscheidet sie von einer Pathologie zweiter Ordnung - der Missachtung zumindest teilweise noch bewusst und adressieren diese auch in ihren Interpretationen. Um beim Beispiel des Zugfahrens zu bleiben, läge eine Pathologie erster Ordnung dann vor, wenn die Kritik der Fahrgäste an den Verdatungspraktiken in Zügen vonseiten der Bahn systematisch ignoriert, kleingeredet oder lächerlich gemacht würde. Damit werden die verdateten Subjekte - die Fahrgäste - auf pathologische Weise in ihrer Standardautorität von den anderen TkR (dem Bahnpersonal) der Verdatungspraxis Videoüberwachung im Zug missachtet. Wenn darüber hinaus ihr Kampf um Anerkennung medial ausgeblendet oder als illegitim (,Verbrecherinnen', ,Terroristinnen') gebrandmarkt würde, stünde auch die Anerkennung als soziale Bewegung in Zweifel, was die Missachtungen als Standardautorität in den Verdatungspraktiken noch verstärkt.

Sozialontologisch lassen sich diese Pathologien entlang der beiden Hinsichten unterscheiden, auf die die Standardautorität wirkt (Abschnitt 2.1): Mit Blick auf die erste Hinsicht werden zum einen die Kontestationen als kompetenter Interpretationsversuch der Betroffenen mit Blick auf die Normen von $p$ missachtet, was zum anderen häufig mit dem systematischen Ignorieren der Pflicht zur Rechenschaft über die eigenen Rolleninterpretationen vonseiten der anderen Rollenträgerinnen einhergeht. Mit Blick auf die zweite Hinsicht werden die Betroffenen in ihrer Autorität missachtet, 
die Struktur von $p$ selbst, die Verteilung der Rollenprivilegien und Rollenverpflichtungen, sowie die Frage, welche Rollen überhaupt in $p$ vorkommen sollten, zu interpretieren. In beiden Hinsichten geschieht dies implizit oder explizit mithilfe verschiedener Mechanismen, angefangen vom Ignorieren, Lächerlich-Machen oder dem Klassifizieren von Interpretationsversuchen als ,unsagbar' (epistemic injustice), ${ }^{40}$ über die Polarisierung des Diskurses (othering), ${ }^{41}$ bis hin zu manifester Unterdrückung (oppression). ${ }^{42}$

Für die Klassifizierung als Pathologie ist es unerheblich, ob diese Mechanismen absichtlich eingesetzt werden oder nicht. Entscheidend ist vielmehr, dass die Struktur von $p$, also die sozialen Beharrungskräfte und Machtasymmetrien in den Rollenstatus, erheblich dazu beiträgt, dass der Anspruch der Betroffenen auf Anerkennung als Standardautorität andauernd und umfassend frustriert wird. So wurde beispielsweise zu Beginn der Fridays-for-Future-Proteste der massenhafte (Aufruf zum) Verstoß gegen die allgemeine Schulpflicht thematisiert. Gerade in Kombination mit einer (liberalen) Demokratievorstellung, nach der demokratische Interventionen immer legal sein müssen, ließ sich dieser Verstoß gut zur Kritik instrumentalisieren - speziell von Akteuren, die aufgrund ihrer Machtposition innerhalb des politischen und rechtlichen Systems besonders glaubwürdig Gesetzestreue anmahnen können.

Für die normative Wirkmächtigkeit derartiger Missachtungen waren im vorliegenden Beispiel also strukturelle Gründe wie beispielsweise Normen innerhalb von Praktiken politischen Protests sowie stark asymmetrische Rollenstatus zwischen Befürworterinnen und Gegnerinnen hochgradig mitentscheidend. Mit Blick auf momentane Verdatungspraktiken lässt sich leicht ersehen, wie insbesondere die Big Nine (Google, Microsoft, Apple, Facebook, IBM, Amazon, Tencent, Alibaba, Baidu) ${ }^{43}$ ihr Marktmonopol und ihre damit einhergehende Machtstellung funktionalisieren, um die Interpretationen der Verdatungssubjekte systematisch zu ignorieren. So werden die datenökonomischen Aggregations- und Monetarisierungsstrategien mit dem Verweis auf Geschäftsgeheimnisse möglichst im Dunkeln gehalten, Datenschutzerklärungen und AGBs unverständlich bis an die Grenze des Zulässigen verfasst, Auskunfts- und Löschpflichten nur sehr zögerlich und nach mehrmaligen Aufforderungen befolgt, Konzepte der

40 Vgl. Fricker (2007); Kidd et al. (2017).

41 Vgl. Spivak (1985); Reuter (2002).

42 Vgl. Young (2004); Haslanger (2017).

43 Vgl. Webb (2019). 
Zweckbindung und Datenminimierung so weit als möglich unterlaufen und IT-Sicherheitsbestimmungen missachtet. ${ }^{44}$

Die hohe Informationsasymmetrie vieler Verdatungspraktiken beschert den Verdatungsinstanzen zudem ein erhebliches Herrschaftswissen, das sich in den genannten Beispielen als strukturelle Missachtung der Standardautorität der Verdatungssubjekte in der ersten Hinsicht manifestiert. Unterstützt wird dies in vielen Fällen durch eine neoliberale Ausformulierung der datenökonomischen Zusammenhänge, die das Verdatungssubjekt als Eigentümerin ihrer Daten sieht und so der Ökonomisierung der Datenaggregation und der Werbungspersonalisierung das Wort redet. ${ }^{45}$ Diese bauen auf einer normativen Struktur auf, welche wiederum von einer individualistischen Vorstellung von Autonomie als ,self-ownership geprägt ist. ${ }^{46}$

An dieser Stelle geht die Verweigerung der Anerkennung als Standardautorität von der ersten in die zweite Hinsicht über, d. h. betreffend die Interpretation und Kontestation der Struktur von $p_{v}$ selbst. Besonders klar zeigt sich das Wechselspiel zwischen individualistischer Autonomievorstellung und neoliberaler Datenökonomie in dem Datenschutzkonzept der Einwilligung. In Übereinstimmung mit diesen beiden normativen Strukturierungsprinzipien von Verdatungspraktiken wird hier suggeriert, dass, sofern die Verdatungssubjekte in einer wohlinformierten Entscheidung in die Verdatung einwilligen (informed consent) ${ }^{47}$, die Verdatung zulässig sein müsse, da sie Ausdruck von ,Autonomie‘ und ,Eigentümerschaft' sei.

Eine solche wohlinformierte Einwilligung erfordert jedoch ein hohes $\mathrm{Maß}$ an ökonomischem wie technischem Vorwissen, über das die meisten Verdatungssubjekte nicht verfügen dürften. Darüber hinaus wird sie durch eine Reihe von epistemischen Hürden erschwert, wie beispielsweise der fehlenden Transparenz in der Entscheidungsstruktur von Algorithmen, ${ }^{48}$ den für die Nutzenden häufig unverständlichen AGBs, ${ }^{49}$ der Diskrepanz zwischen einmaliger Einwilligung und kontinuierlicher Datenerhebung, der oftmals den Nutzenden nicht präsenten Möglichkeit der Aggregation zwischen Zugangs- und Inhaltsdaten im Sinne der Deanonymisierung, ${ }^{50}$

44 Vgl. Brignull (2013); Calo (2014); Nadler/McGuigan (2018); Litman-Navarro (12.06.2019).

45 Vgl. Zuboff (2019).

46 Vgl. Mill (1859: S. 22).

47 Vgl. Dworkin (1988: Kap. 7); Maclean (2009).

48 Vgl. Barocas et al. (2019).

49 Vgl. Litman-Navarro (12.06.2019).

50 Vgl. Narayanan/Shmatikov (2008); Rocher et al. (2019). 
sowie der Unvorhersehbarkeit zukünftiger Nutzungsmöglichkeiten für dauerhaft gespeicherte Daten. ${ }^{51}$

Vor diesem Hintergrund werden die verdateten Subjekte von den Verdatungsinstanzen zwar vordergründig als Standardautoritäten anerkannt, indem einerseits mit der Figur der Einwilligung ihre Performanzen scheinbar als kompetente Interpretationen von $p_{v}$ gewürdigt werden. Gleichzeitig werden ihre Einwilligungen in die Verdatung auch als Ausdruck der Zustimmung zu den Praxisnormen und der Verteilung der Rollenstatus interpretiert. Dabei wird jedoch häufig in unredlicher Weise unterschlagen, dass die Verdatungssubjekte einerseits in vielen Fällen gar keine andere Wahl haben, als den Datenschutzerklärungen und AGBs zuzustimmen, um ihre soziale Teilhabe zu sichern und bestimmte Dienste nutzen zu können. Andererseits verlangen die oben angesprochenen epistemischen Hürden Kompetenzen, die über eine grundsätzliche, digital literacy` weit hinausgehen. ${ }^{52}$ Diese Kompetenzen bringt bei weitem nicht jedes Verdatungssubjekt mit. Den Verdatungssubjekten die Möglichkeit zu geben, in die Verdatung einzuwilligen, markiert daher keine Anerkennung als Standardautorität, sondern vielmehr eine Pseudo-Anerkennung als, super-skilled performer ${ }^{6}{ }^{53}$ Sie ist der schlecht getarnte Versuch, mithilfe der asymmetrischen Machtstruktur in $p_{v}$ rechtliche Mindestanforderungen auf eine Weise zu erfüllen, die die Verwertungslogik der Datenökonomie nicht antastet. $^{54}$

Dennoch zeigen die immer wieder aufflammende Kritik sowie die Versuche, einzelne Verdatungsinstanzen zur Rechenschaft zu ziehen, dass an vielen Stellen ein Bewusstsein für die strukturelle Missachtung besteht oder entsteht. ,Grassroots-Bewegungen' und NGOs fordern informationelle Selbstbestimmung ein, Medien thematisieren Datenschutzskandale, zumindest Teile einer aufgeklärten Öffentlichkeit sind sich inzwischen der Risiken und Probleme eines allzu leichtfertigen Umgangs mit den eigenen Daten bewusst. Die „Übersetzungsfunktion" ${ }^{55}$ dieser Öffentlichkeiten sorgte in der Vergangenheit dafür, dass politische Akteure sich zumindest des Themas angenommen haben; Enthüllungen von Whistleblowern wie bei-

51 Vgl. Kriesel (2016).

52 Vgl. Bonfadelli (2004); Jenkins et al. (2009); Hagendorff (2018).

53 Ich verdanke diesen Begriff einer Diskussion mit Thomas Renkert. Für eine genauere Auseinandersetzung mit dem Problem vgl. Loh (2019b).

54 Vgl. Zuboff (2019).

55 Habermas (2008: S. 150); Loh (2020: S. 167). 
spielsweise Edward Snowden haben ihr Übriges dazu getan, dass unter anderem die EU-Verhandlungen zur DSGVO letztlich erfolgreich waren. ${ }^{56}$

Sobald aber eine strukturelle Missachtung der Standardautorität bestimmter Rollen in $p_{v}$,aus systematischen Gründen nicht als expliziter Konflikt um Normen" 57 erkannt und geführt werden kann, handelt es sich um eine Pathologie zweiter Ordnung. Den Betroffenen ist entweder nicht bewusst, dass sie nicht als Standardautorität anerkannt werden, oder aber sie sind nicht in der Lage, ihre diffusen Missachtungserfahrungen als Kritik an der Struktur der Praxis zu formulieren. Bei einer Pathologie zweiter Ordnung verdecken die jeweiligen Praktiken „den Umstand ihres ,Gemachtseins ${ }^{6}$, den Umstand also, dass sie Resultat menschlicher Praxis, das heißt Resultat kollektiver Instituierung und Akzeptanz ${ }^{\text {“58 }}$ sind. Dies geschieht häufig mittels Mechanismen der Essentialisierung/Naturalisierung bzw. Verdinglichung. ${ }^{59}$ Die Praxis bzw. einige ihrer Normen treten ihren Akteuren wie ein Objekt oder Naturereignis gegenüber, das eine eigene Essenz besitzt und nicht sozial bedingt ist. Damit einher geht oft eine Vorstellung von Unveränderbarkeit und Alternativlosigkeit, umso mehr, als es sich in den Fällen von Verdatungspraktiken zumeist um technologische Entwicklungen und Veränderungen handelt, die oftmals deterministisch betrachtet werden. ${ }^{60}$ Mit diesen Verdeckungsmechanismen wird die Kritik an der Struktur der Praxis verunmöglicht und damit die Anerkennung als Standardautorität verweigert:

Wer soziale Institutionen oder Regeln so behandelt oder wahrnimmt, als hätten sie einen rein sachlichen, unveränderbaren Charakter, begeht einen Kategorienfehler und nimmt sich selbst die Chance, diese sozialen Verhältnisse zu verändern oder zu verbessern. ${ }^{61}$

Auch in Verdatungspraktiken kommen diese Verdeckungsmechanismen zunehmend zum Vorschein, beispielsweise in Form der Resignation vor dem Hintergrund einer immer umfassenderen Aggregierung und Moneta-

56 Vgl. hierzu den Film „Democracy - Im Rausch der Daten“ von David Bernet (2015).

57 Stahl (2013: S. 367; Herv. entfernt; W. L.).

58 Jaeggi (2009: S. 542).

59 Vgl. Lukács (1923); Adorno/Horkheimer (1969). Zu einer jüngeren Reorientierung des Verdinglichungsbegriffs vgl. Quadflieg (2019).

60 Ich danke Martin Hennig für den Hinweis auf den Technikdeterminismus. Im Bereich der Neoliberalismuskritiken hat sich hier auch der Begriff TINA (There Is No Alternative) eingebürgert.

61 Stahl (2013: S. 422 f.). 
risierung persönlicher Daten einerseits, sowie staatlichen Überwachungstendenzen andererseits. Aussagen wie „Ich habe ja nichts zu verbergen“ lassen sich als Ausdruck von Rationalisierungsbemühungen im Angesicht von als alternativlos und unveränderlich erfahrener Verdatungsstrukturen lesen. Gleichzeitig nehmen die Abgesänge auf liberal-bürgerliche Vorstellungen von Privatsphäre zugunsten einer ,Transparenzgesellschaft ${ }^{62} \mathrm{zu}$, in denen die Befürworterinnen versuchen, die Vorteile einer Post-PrivacyÄra ${ }^{63}$ zu skizzieren.

In dieser Gemengelage kann das sogenannte Privacy Paradox als bekanntes Beispiel einer Pathologie zweiter Ordnung dienen. Kurz gesagt ist damit die Diskrepanz zwischen der Wichtigkeit der eigenen Privatsphäre, die Nutzerinnen immer wieder betonen, und der scheinbaren Sorglosigkeit, mit der sie ihre Daten in Verdatungskontexten freiwillig preisgeben, gemeint. ${ }^{64}$ Diese Diskrepanz ist verschiedentlich erklärt worden, beispielsweise mit kognitiven bzw. psychomotivationalen Effekten wie "cognitive overload" und „ego depletion“, 65 Diskontierungseffekten oder dem ,zeroprice-effect ${ }^{6}{ }^{66}$ wie sie aus der Verhaltensökonomie bekannt sind, oder auch mit kognitiven Dissonanzen zwischen der Bürgerinnen- und der Nutzerinnen-Rolle. ${ }^{67}$ Für die Charakterisierung der Phänomene rund um das Privacy Paradox als Pathologie zweiter Ordnung ist entscheidend, dass hier - teilweise bewusst, teilweise unbewusst - Verdatungspraktiken für die Betroffenen auf eine Weise unverfügbar gemacht werden, dass sie diesen Vorgang nicht mehr wahrnehmen. Sie werden nicht mehr als Standardautorität anerkannt, bemerken dies jedoch nicht. Vielmehr gerieren sich die Verdatungspraktiken als unveränderliche Tatsache, als Ding, dessen Struktur notwendig so und nicht anders sein muss.

Beispielsweise stellt sich die Bezahlung vermeintlich kostenloser Apps und stark rabattierter Hardware mit dem Nutzungsverhalten und den persönlichen Daten der Verdatungssubjekte diesen als unumgänglicher Marktmechanismus der Datenökonomie dar, welcher sich wiederum alternativlos in eine gesamtkapitalistische Verwertungslogik einfügt. ${ }^{68}$ Die Fra-

62 Vgl. Brin (1998). Eine solche skeptisch-affirmative Sichtweise wird wohlgemerkt von einer Vielfalt kritisch-dystopischer Perspektivierungen der Idee der Transparenzgesellschaft kontrastiert. Vgl. hierzu statt vieler Han (2012); Eggers (2014).

63 Vgl. Heller (2011).

64 Vgl. Barnes (2006); Taddicken (2014); Kokolakis (2017).

65 Veltri und Ivchenko (2017: S. 240).

66 Vgl. Shampanier et al. (2007).

67 Vgl. Kneip (2010).

68 Vgl. Staab (2019). 
ge, ob das Monetarisierungsmodell der Datenökonomie überhaupt eine wünschbare ökonomische Praxis darstellt, ${ }^{69}$ wird außerhalb akademischer Diskurse nur selten gestellt. Um diesen Diskurs auf die Verdatungssubjekte - also uns alle - zu erweitern und unsere informationelle Privatheit als Standardautoritäten in unseren Verdatungspraktiken zu sichern, müssen diese Pathologien informationeller Privatheit benannt, reflektiert und aufgelöst werden. Dazu bedarf es einer noch genaueren sozialtheoretischen Analyse dieser Praktiken, als ich es hier zu leisten vermag, und einer darauffolgenden sozialtheoretisch informierten Kritik der Praktiken als pathologisch.

\section{Schluss}

Wie sich gezeigt hat, lässt sich informationelle Privatheit mittels des SAMP auf einer sozialontologisch fundamentalen Ebene als wechselseitige Anerkennung als Standardautorität in Verdatungspraktiken beschreiben. Die Vorteile einer solchen Beschreibung liegen vor allem in der normativen Sparsamkeit, mit der das SAMP Verdatungspraktiken als defizitär bzw. pathologisch beschreiben kann: In ihnen werden systematisch (anhaltend und umfassend) und aus strukturellen Gründen die Trägerinnen der Rolle Verdatungssubjekt nicht als Standardautoritäten anerkannt. Damit geht das SAMP über die von Nissenbaum postulierte ,Angemessenheit' von Verdatungskontexten und den ,appropriate flow of information' insofern hinaus, als es diese rein praxisinternen Angemessenheitsansprüche um eine zweite, sozialontologische Ebene - die Ebene der Standardautorität - ergänzt.

Gleichzeitig bedient sich das SAMP aber auch keines externen Maßstabes von Privatheit und informationeller Selbstbestimmung, wie er oftmals in moralisch-deduktiv argumentierten Privatheitstheorien in Anschlag gebracht wird. Vielmehr nimmt das SAMP mit der Konzeption von sozialen Pathologien der Privatheit die Vorstellung in den Blick, dass auch Verdatungspraktiken als soziale Gebilde stabilisiert und reproduziert werden müssen - und dass diese Stabilisierung und Reproduktion an der Zustimmung der Praxisteilnehmenden hängt, die diese performativ in ihren Rollen zum Ausdruck bringen.

Durch dieses Ausüben ihrer Rolle(n) reproduzieren sie nicht nur die (Verdatungs)praxis, sondern verändern diese auch durch die Interpretati- 
on-qua-Performanz ihrer Rollenverpflichtungen. Ohne die wechselseitige Anerkennung als kompetente Performerinnen (und damit als Standardautorität), gehen diese Interpretationen verloren, Praktiken verhärten, verlieren die performative Zustimmung und werden pathologisch. In diesem Text habe ich lediglich die Möglichkeit von Pathologien informationeller Privatheit diskutiert und einige sehr knappe Beispiele dafür gegeben. Ziel muss es jedoch sein, mithilfe dieser Pathologiediagnose dazu beizutragen, heutige Verdatungspraktiken zu transformieren. In diesem Sinne dient die Analyse von Verdatungspraktiken als pathologisch nicht nur einem akademischen Selbstzweck, in bester Manier der Kritischen Theorie ist sie vielmehr „als Analyse Kritik“70.

\section{Literaturverzeichnis}

Adler, Emanuel/Pouliot, Vincent (2011): „International practices“. In: International Theory 3 (1), S. 1-36.

Adorno, Theodor W./Horkheimer, Max (1969): „Dialektik der Aufklärung“. In: Schmidt, Alfred (Hrsg.): Gesammelte Schriften. Frankfurt am Main: Fischer, S. 13-292.

Allen, Anita (1988): Uneasy access: Privacy for women in a free society. Totowa NJ: Rowman \& Littlefield.

Barnes, Susan (2006): „A privacy paradox: Social networking in the United States“. In: First Monday 11 (9). URL: https://firstmonday.org/ojs/index.php/fm/article/ view/1394/1312 [Abruf am: 02.12.2020].

Barocas, Solon et al. (2019): Fairness and machine learning: Limitations and Opportunities. URL: https://fairmlbook.org/ [Abruf am: 19.05.2019].

Berger, Peter L./Luckmann, Thomas (1969): Die gesellschaftliche Konstruktion der Wirklichkeit. Frankfurt am Main: Fischer.

Bonfadelli, Heinz (2004): Medienkompetenz und Medienleistungen in der Informationsgesellschaft. Zürich: Pestalozzianum.

Brignull, Harry (2013): $90 \%$ of Everything. URL: https://www.90percentofeverything.com/2013/07/23/the-slippery-slope/ [Abruf am: 19.01.2020].

Brin, David (1998): The transparent society. Reading MA: Perseus Books.

Calo, Ryan (2014): „Digital Market Manipulation“. In: The George Washington Law Review 82 (4), S. 995-1051.

Celikates, Robin (2009): Kritik als soziale Praxis. Frankfurt am Main: Campus.

Dienlin, Tobias/Trepte, Sabine (2015): „Is the privacy paradox a relic of the past?“. In: European Journal of Social Psychology 45 (3), S. 285-297. 
Dworkin, Gerald (1988): The theory and practice of autonomy. Cambridge: Cambridge University Press.

Eggers, Dave (2014): The Circle. London: Penguin Books.

Fricker, Miranda (2007): Epistemic injustice: Power and the ethics of knowing. Oxford: Oxford University Press.

Fried, Charles (1984): „Privacy: A moral analysis“. In: Schoeman, Ferdinand (Hrsg.): Philosophical dimensions of privacy. Cambridge: Cambridge University Press, S. 203-222.

Gavison, Ruth (1980): „Privacy and the Limits of Law“. In: The Yale Law Journal 89 (3), S. 421-471.

Goffman, Erving (1969): Wir alle spielen Theater: Die Selbstdarstellung im Alltag. München: Piper.

Habermas, Jürgen (2008): „Hat die Demokratie noch eine epistemische Funktion?“. In: Ders. (Hrsg.): Ach, Europa. Frankfurt am Main: Suhrkamp, S. 138-191.

Hagendorff, Thilo (2018): „Übersehene Probleme des Konzepts der Privacy Literacy“. In: Roßnagel, Alexander et al. (Hrsg.): Die Fortentwicklung des Datenschutzes. Wiesbaden: Springer Vieweg, S. 99-120.

Han, Byung-Chul (2012): Transparenzgesellschaft. Berlin: Matthes \& Seitz.

Haslanger, Sally (2000): „Gender and Race: (What) Are They? (What) Do We Want Them To Be?““. In: Noûs 34 (1), S. 31-55.

Haslanger, Sally (2005): „What Are We Talking about? The Semantics and Politics of Social Kinds“. In: Hypatia 20 (4), S. 10-26.

Haslanger, Sally (2017): „Objectivity, Epistemic Objectification, and Oppression“. In: Kidd, Ian James et al. (Hrsg.): The Routledge handbook of epistemic injustice. Milton: Taylor and Francis, S. 279-290.

Heller, Christian (2011): Post Privacy: Prima leben ohne Privatsphäre. München: C.H. Beck.

Honneth, Axel (2000): „Pathologien des Sozialen“. In: Ders. (Hrsg.): Das Andere der Gerechtigkeit. Frankfurt am Main: Suhrkamp, S. 11-69.

Honneth, Axel (2003): „Umverteilung als Anerkennung: Eine Erwiderung auf Nancy Fraser“. In: Fraser, Nancy/Honneth, Axel (Hrsg.): Umverteilung oder Anerkennung? Frankfurt am Main: Suhrkamp, S. 129-224.

Honneth, Axel (2011): Verwilderungen des sozialen Konflikts. Max-Planck-Institut für Gesellschaftsforschung (MPIfG Working Paper: 11/4). URL: http://www.mpi fg.de/pu/workpap/wp11-4.pdf [Abruf am: 01.06.2011].

Honneth, Axel (2014): „Die Krankheiten der Gesellschaft: Annäherung an einen nahezu unmöglichen Begriff“. In: WestEnd 1, S. 45-60.

Jaeggi, Rahel (2009): „Was ist eine (gute) Institution?“. In: Forst, Rainer et al. (Hrsg.): Sozialphilosophie und Kritik. Frankfurt am Main: Suhrkamp, S. 528544.

Jaeggi, Rahel (2014): Kritik von Lebensformen. Berlin: Suhrkamp.

Jaeggi, Rahel (2018): „Replik-Replik: Ökonomie als soziale Praxis“. In: Zeitschrift für Wirtschafts- und Unternehmensethik 19 (3), S. 442-466. 
Jenkins, Henry et al. (2009): Confronting the Challenges of Participatory Culture. London: MIT Press.

Kidd, Ian James et al. (Hrsg.) (2017): The Routledge Handbook of Epistemic Injustice. Milton: Taylor and Francis.

Kneip, Veronika (2010): Consumer Citizenship und Corporate Citizenship. BadenBaden: Nomos.

Kokolakis, Spyros (2017): „Privacy attitudes and privacy behaviour: A review of current research on the privacy paradox phenomenon“. In: Computers \& Security 64 , S. 122-134.

Kriesel, David (2016): SpiegelMining: Wer, wann, was, mit wem?: Das soziale Netz der SpiegelOnline-Redakteure. URL: http://www.dkriesel.com/blog/2016/0814_ spiegelmining_soziales_netz_redakteure [Abruf am: 14.04.2020].

Litman-Navarro, Kevin (2019): „We Read 150 Privacy Policies. They Were an Incomprehensible Disaster“. In: New York Times (12.06.2019). URL: https:// www.nytimes.com/interactive/2019/06/12/opinion/facebook-google-privacy-policies.html [Abruf am: 16.09.2019].

Loh, Wulf (2017): „Literaturwissenschaft als Sozialkritik“. In: Albrecht, Andrea/ Schramm, Moritz/Venzl, Tilman (Hrsg.): Literatur und Anerkennung. Wien: Lit Verlag, S. 159-184.

Loh, Wulf (2018): „A Practice-Theoretical Account of Privacy“. In: Ethics and Information Technology 20 (4), S. 233-247.

Loh, Wulf (2019a): Legitimität und Selbstbestimmung: Eine normative Rekonstruktion des Völkerrechts. Baden-Baden: Nomos.

Loh, Wulf (2019b): „Sozialontologische Bedingungen von Privatheit“. In: Behrendt, Hauke et al. (Hrsg.): Privatsphäre 4.0. Stuttgart: Metzler, S. 33-53.

Loh, Wulf (2020): „Informationelle Privatheit als Bedingung für Demokratie“. In: Archiv für Rechts- und Sozialphilosophie 162, S. 165-181.

Lukács, Georg (1923): „Die Verdinglichung und das Bewußtsein des Proletariats“. In: Ders. (Hrsg.): Geschichte und Klassenbewußtsein. Berlin: Malik, S. 97-192.

Maclean, Alasdair (2009): Autonomy, informed consent and medical law: A relational challenge. Cambridge: Cambridge University Press.

Mead, George Herbert (1934): Mind, Self, and Society. Hg. von Charles Morris. Chicago: Chicago University Press.

Mill, John Stuart (1859): On Liberty. London: Longmans.

Moor, James (1990): „The Ethics of Privacy Protection“. In: Library Trends 39 (1 \& 2), S. 69-82.

Moor, James (1997): „Towards a Theory of Privacy in the Information Age“. In: Computers and Society 27 (3), S. 27-32.

Nadler, Anthony/McGuigan, Lee (2018): „An impulse to exploit: the behavioral turn in data-driven marketing“. In: Critical Studies in Media Communication 35 (2), S. 151-165.

Narayanan, Arvind/Shmatikov, Vitaly (2008): Robust De-anonymization of Large Sparse Datasets. 2008 IEEE Symposium on Security and Privacy, S. 111-125. 
Nissenbaum, Helen (2004): „Privacy as Contextual Integrity“. In: Washington Law Review 79 (1), S. 119-158.

Nissenbaum, Helen (2010): Privacy in context: Technology, policy, and the integrity of social life. Stanford CA: Stanford Law Books.

Parent, William A. (1983): „Recent Work on the Concept of Privacy“. In: American Philosophical Quarterly 20 (4), S. 341-355.

Quadflieg, Dirk (2019): Vom Geist der Sache: Zur Kritik der Verdinglichung. Frankfurt am Main: Campus.

Rawls, John (2002): Das Recht der Völker. Berlin: De Gruyter.

Reuter, Julia (2002): Ordnungen des Anderen. Bielefeld: transcript.

Rocher, Luc et al. (2019): „Estimating the success of re-identifications in incomplete datasets using generative models“. In: Nature Communications 10 (1), S. 3069-3078.

Roessler, Beate/Mokrosinska, Dorota (2013): „Privacy and social interaction“. In: Philosophy \& Social Criticism 39 (8), S. 771-91.

Rössler, Beate (2001): Der Wert des Privaten. Frankfurt am Main: Suhrkamp.

Rössler, Beate (2015): „Should personal data be a tradable good? On the moral limits of markets in privacy“. In: Roessler, Beate/Mokrosinska, Dorota (Hrsg.): Social dimensions of privacy. Cambridge: Cambridge University Press, S. 141-161.

Ryle, Gilbert (2009): The concept of mind. London und New York: Routledge.

Searle, John (1969): Speech acts: An essay in the philosophy of language. Cambridge: Cambridge University Press.

Shampanier, Kristina et al. (2007): „Zero as a Special Price: The True Value of Free Products“. In: Marketing Science 26 (6), S. 742-757.

Spivak, Gayatri (1985): „The Rani of Sirmur: An Essay in Reading the Archives“. In: History and Theory 24 (3), S. 247-272.

Staab, Philipp (2019): Digitaler Kapitalismus. Markt und Herrschaft in der Ökonomie der Unknappheit. Frankfurt am Main: Suhrkamp.

Stahl, Titus (2013): Immanente Kritik: Elemente einer Theorie sozialer Praktiken. Frankfurt am Main: Campus.

Strawson, Peter (1962): Freedom and resentment and other essays. London: Routledge.

Taddicken, Monika (2014): „The,Privacy Paradox“ in the Social Web“. In: Journal of Computer-Mediated Communication 19 (2), S. 248-273.

Tavani, Herman (2007): „Philosophical theories of privacy: Implications for an adequate online privacy policy“. In: Metaphilosophy 38 (1), S. 1-22.

Veltri, Giuseppe A./Ivchenko, Andriy (2017): „The impact of different forms of cognitive scarcity on online privacy disclosure“. In: Computers in Human Behavior 73, S. 238-246.

Webb, Amy (2019): The Big Nine: How the tech titans and their thinking machines could warp humanity. New York: PublicAffairs.

West, Candace/Zimmerman, Don (1987): „Doing Gender“. In: Gender \& Society 1 (2), S. 125-151. 
Westin, Alan (1967): Privacy and freedom. New York: Atheneum Press.

Young, Iris Marion (2004): „Five Faces of Oppression“. In: Heldke, Lisa M./ O'Connor, Peg (Hrsg.): Oppression, privilege, and resistance. Boston: McGrawHill, S. 38-65.

Zuboff, Shoshana (2019): The age of surveillance capitalism: The fight for the future at the new frontier of power. London: Profile Books.

Zurn, Christopher (2011): „Social Pathologies as Second-Order Disorders“. In: Petherbridge, Danielle (Hrsg.): Axel Honneth: Critical essays. Boston: Brill, S. 345-370. 
